Article

\title{
Expression of Insulin Receptor and c-MET Is Associated with Clinicopathologic Characteristics and Molecular Subtypes in Premenopausal Breast Cancer Patients
}

\author{
Nehad M. Ayoub ${ }^{1, *(\mathbb{C}}$, Rami J. Yaghan ${ }^{2}{ }^{(}$, Alia H. Al-Mohtaseb ${ }^{3}{ }^{(}$, Najla Aldaoud $^{3}{ }^{(}$, \\ Ismail I. Matalka ${ }^{3}$ and Muwada E. Elhassan ${ }^{3}$ \\ 1 Department of Clinical Pharmacy, Faculty of Pharmacy, Jordan University of Science and Technology, \\ Irbid 22110, Jordan \\ 2 Department of Surgery, College of Medicine and Medical Sciences, Arabian Gulf University, \\ Manama P.O. Box 26671, Bahrain; ramijy@agu.edu.bh \\ 3 Department of Pathology and Microbiology, Faculty of Medicine, Jordan University of Science and \\ Technology, Irbid 22110, Jordan; ahmohtaseb@just.edu.jo (A.H.A.-M.); nhaldaoud2@just.edu.jo (N.A.); \\ Imatalka@just.edu.jo (I.I.M.); muwada.sir@gmail.com (M.E.E.) \\ * Correspondence: nmayoub@just.edu.jo; Tel.: +962-796-855-653
}

Received: 9 January 2020; Accepted: 24 February 2020; Published: 29 February 2020

\begin{abstract}
Receptor Tyrosine Kinases (RTKs) represent a class of transmembrane receptors known to play an important role in cancer development and progression. In this study, the expression of insulin receptor (IR) and the hepatocyte growth factor receptor (c-MET) was examined in breast cancer patients. Immunohistochemistry for IR and c-MET was performed on 71 cases of invasive breast cancer and expression scores were correlated with clinicopathologic characteristics and molecular subtypes and further stratified based on a menopausal status. Expression of IR was significantly associated with the tumor grade $(p=0.017)$ and estrogen receptor $(\mathrm{ER})$ expression $(p=0.015)$. There was a significant positive correlation between IR and c-MET expression scores (rho $=0.458, p<0.001$ ). Among premenopausal cases, IR scores were significantly higher in patients with grade I/II disease $(p=0.025)$, ER-positive $(p=0.030)$, and progesterone receptor $(\mathrm{PR})$-positive carcinoma $(p=0.015)$. $c-M E T$ expression scores were significantly higher among premenopausal patients with ER-positive $(p=0.007)$ and PR-positive carcinoma $(p=0.024)$. IR expression scores were significantly different among molecular subtypes for all patients $(p=0.006)$ and among premenopausal cases $(p=0.035)$. $c-M E T$ expression was statistically different among molecular subtypes for premenopausal patients $(p=0.019)$. Survival analysis revealed that the expression status of IR and c-MET was not associated with overall survival. Our findings support a favorable prognostic value for IR and c-MET expression in premenopausal breast cancer patients.
\end{abstract}

Keywords: breast cancer; receptor tyrosine kinases; insulin receptor; c-MET; immunohistochemistry; menopausal status

\section{Introduction}

Receptor tyrosine kinases (RTKs) are transmembrane receptors known to bind growth factors mediating the integration and interpretation of diverse extracellular stimuli and functions [1]. Historically, RTKs define the prototypical class of oncogenes involved in most forms of human malignancies [2]. Deregulations of RTKs in terms of mutations or aberrant expression enhance cancer cell proliferation, survival, and invasion, which ultimately drive the development of aggressive tumor phenotypes [3]. 
The growth of breast cancer cells is under coordinate regulation of growth factors and hormones acting through their respective receptors [1,4]. Multiple RTKs were identified for their oncogenic potential in breast cancer. Insulin receptor (IR) is an RTK increasingly recognized to be involved in cancer development and progression [5]. Accumulating evidence revealed a role for insulin and insulin-like growth factors in development and progression of breast cancer [6-8]. Overexpression of IR was reported in human breast cancer tissues [7,9-11]. In a mouse model of type II diabetes, IR promoted breast cancer mitogenesis and hyperinsulinemia accelerated the progression of mammary tumors [12]. Insulin resistance and hyperinsulinemia have been shown to drive mitogenic tumor growth and increase cell proliferation and migration through activation of IR signaling [5,13-15]. In line with this, conditions associated with insulin resistance such as type II diabetes mellitus, obesity, and metabolic syndrome are now recognized as important risk factors for the development and progression of a variety of malignancies including breast cancer $[5,13,16]$. Aberrant IR expression has been shown to mediate cancer resistance to both conventional and targeted therapies [17]. Measures to improve insulin resistance and obesity reduce the risk of epithelial cancers. Metformin reduced breast cancer risks by reducing insulin levels [11]. In addition, metformin treatment in breast cancer patients who were not diabetic reduced levels of IR expression in tumors and reduced phosphorylation of several downstream signaling molecules such as protein kinase B (AKT), extracellular signal-regulated kinase 1/2 (ERK1/2), and AMP-activated protein kinase (AMPK) [18]. Despite the availability of strong evidence from preclinical studies to support the oncogenic potential of IR in breast cancer cells, the impact of IR expression on the clinicopathologic characteristics of breast cancer patients is less clear.

The hepatocyte growth factor (HGF) receptor, c-MET, is an RTK known to promote oncogenic transformation and progression of cancers. Activation of c-MET by its natural ligand, HGF, is associated with aggressive tumor growth characterized by cancer cell proliferation, migration, invasion, angiogenesis, and metastasis $[19,20]$. Clinical studies showed that c-MET is overexpressed in $20 \%-30 \%$ of breast cancer cases and is a strong, independent predictor of reduced survival and poor outcomes [21,22]. c-MET expression in breast tissue is variable with the lowest levels in normal breast tissue, higher expression in ductal carcinoma in situ (DCIS), and the highest levels in invasive carcinoma [23]. Despite its oncogenic potential, the impact of c-MET expression on established prognostic factors in breast cancer patients is less established. In addition, most studies evaluated the expression of c-MET in basal-like tumors and little data is available regarding the expression of c-MET in other molecular subtypes. c-MET has been shown to be necessary for an optimal response to insulin in hepatocytes by engaging with IR in a hybrid complex of both receptors [24].

Previous reports had addressed expression of IR and c-MET in breast cancer tissue. However, less data is available on the association between expression levels of these receptors in breast cancer patients. In addition, the impact of IR and c-MET expression on disease prognosis and clinicopathologic characteristics is inconsistent. In this study, the expression of IR and c-MET was evaluated in tumors from primary breast cancer patients. Associations between expression levels of both RTKs with clinicopathologic factors and molecular subtypes were evaluated and further stratified based on menopausal status. These findings are particularly important because effective therapeutic approaches require stratification of patients based on specific tumor markers essential to assess the efficacy of new molecular therapies in terms of disease prevention and/or treatment.

\section{Materials and Methods}

\subsection{Patients and Tumor Samples}

Seventy-one patients with histologically confirmed diagnosis of invasive breast cancer and complete data regarding the receptor status and molecular subtype were retrieved from archives of King Abdullah University Hospital (KAUH) through the period of 2004-2014. All included patients underwent mastectomy or wide local excision with or without chemotherapy, according to tumor board recommendations. Relevant demographic and anthropometric data were collected from medical 
records and the electronic database. Body mass index (BMI) was calculated using the standard method as weight in kilograms divided by the height squared in meters. Based on World Health Organization (WHO) definition for obesity, patients were classified as underweight (BMI $<18.5 \mathrm{~kg} / \mathrm{m}^{2}$ ), normal (BMI $18.5-24.99 \mathrm{~kg} / \mathrm{m}^{2}$ ), overweight (BMI $25.0-29.99 \mathrm{~kg} / \mathrm{m}^{2}$ ), and obese (BMI $\geq 30.0 \mathrm{~kg} / \mathrm{m}^{2}$ ) [25].

Pathological data were retrieved from pathology reports issued by the Pathology Department at KAUH at the time of diagnosis. Pathological data included details of tumor size, grade, histological subtype, ipsilateral axillary lymph node status, and presence/absence of lymphovascular invasion (LVI). In addition, pathology reports provided details for the expression status of estrogen receptor (ER), progesterone receptor (PR), and Human Epidermal Growth Factor Receptor 2 (HER2/neu). For HER2, immunohistochemistry (IHC) scores of 0 or +1 were considered negative while a score of +3 indicated positive staining. For equivocal results of +2 score, fluorescence in situ hybridization (FISH) analysis positive for gene amplification was positive for HER2 expression. ER-positive patients received hormonal treatment and patients overexpressing HER2 received trastuzumab for one year.

The clinicopathological stage of breast cancer was obtained according to the tumor-node-metastasis (TNM) cancer staging systems of the American Joint Committee on Cancer (AJCC) [26]. The grade of carcinoma was determined based on the Nottingham Combined Histologic Grade system [27]. Accordingly, tumors were classified into grade I (well-differentiated/low grade), grade II (moderately-differentiated/intermediate grade), and grade III (poorly-differentiated/high grade) carcinomas. Breast cancer was further classified into subtypes based on the expression of hormone receptors and HER2 status [28,29] including luminal (ER+ and/or PR+, HER2+/-), HER2-positive (ER-, PR-, HER2+), and triple-negative (ER-, PR-, HER2-).

The Institutional Review Board (IRB) committee of JUST and KAUH (Research number 33/80/2014) approved the study.

\subsection{Immunohistochemistry}

Immunohistochemistry (IHC) was performed using a Dako autostainer-Plus (Dako, Denmark) following a standard protocol. Paraffin-embedded tissues for each tumor were obtained from the Pathology Department and sections were cut at a thickness of $4 \mu \mathrm{m}$ and prepared for IHC. The sections were dewaxed in two changes in xylene, rehydrated through a descending graded series of ethanol $(100 \%, 90 \%, 80 \%, 70 \%$, and $50 \%)$, and, lastly, rehydrated in distilled water. Antigen retrieval was performed in a PT-link Instrument (Dako, Glostrup, Denmark) using high pH citrate buffer for $20 \mathrm{~min}$. Endogenous peroxidase activity was blocked by incubation with $2.5 \%$ hydrogen peroxide for $10 \mathrm{~min}$. Sections were next washed with phosphate buffered saline (PBS) twice and incubated with primary antibodies for IR (Insulin RB H-70, Santa Cruz Biotechnology, Santa Cruz, CA, USA) diluted 1:100 and c-MET (Met C-12: sc-10, Santa Cruz Biotechnology, Santa Cruz, CA, USA) diluted 1:200 for 45 min at room temperature. These primary antibodies were polyclonal and unconjugated according to the manufacturer's specification sheets. Slides were then washed twice with PBS and signal detection was carried out using The Envision Dual Link System HRP Kit, K8000 (Dako, Glostrub, Denmark) in which slides were incubated with HRP-conjugated secondary antibodies for $30 \mathrm{~min}$ at room temperature. Then, slides were washed and incubated with DAB (3,3'-Diaminobenzine) chromogen for 3-4 min. Lastly, slides were washed with distilled water and counterstained with Mayer's Haematoxylin.

\subsection{Evaluation of Immunostaining}

Immunostaining was evaluated using the immunoreactive score (IRS) system [30,31]. IRS gives a range of $0-12$ as a product of multiplication between a positive cells proportion score (0-4) and a staining intensity score (0-3). Staining extent was scored as 0 (no positive cells), $1(<10 \%), 2(10 \%-50 \%)$, $3(51 \%-80 \%)$, and $4(>80 \%)$ according to the percentage of positively stained cells. Staining intensity was scored as 0 (no color reaction), 1 (mild), 2 (moderate), and 3 (intense). Based on final IRS scores, immunostaining was determined to be negative (IRS score $0-1$ ), mild (IRS score 2-3), moderate (IRS score 4-8), and strongly positive (IRS score 9-12) [30,31]. Immunostaining was evaluated by two 
pathologists (AM and NA) who were blind to patient demographic data, clinical data, and pathological data. The concordance rate was more than 90\%. Discrepancies were resolved by joint discussion. Representative examples of staining for each antibody are shown in Figure 1. Positive control slides were included, and negative controls were run with the primary antibody replaced by buffer.
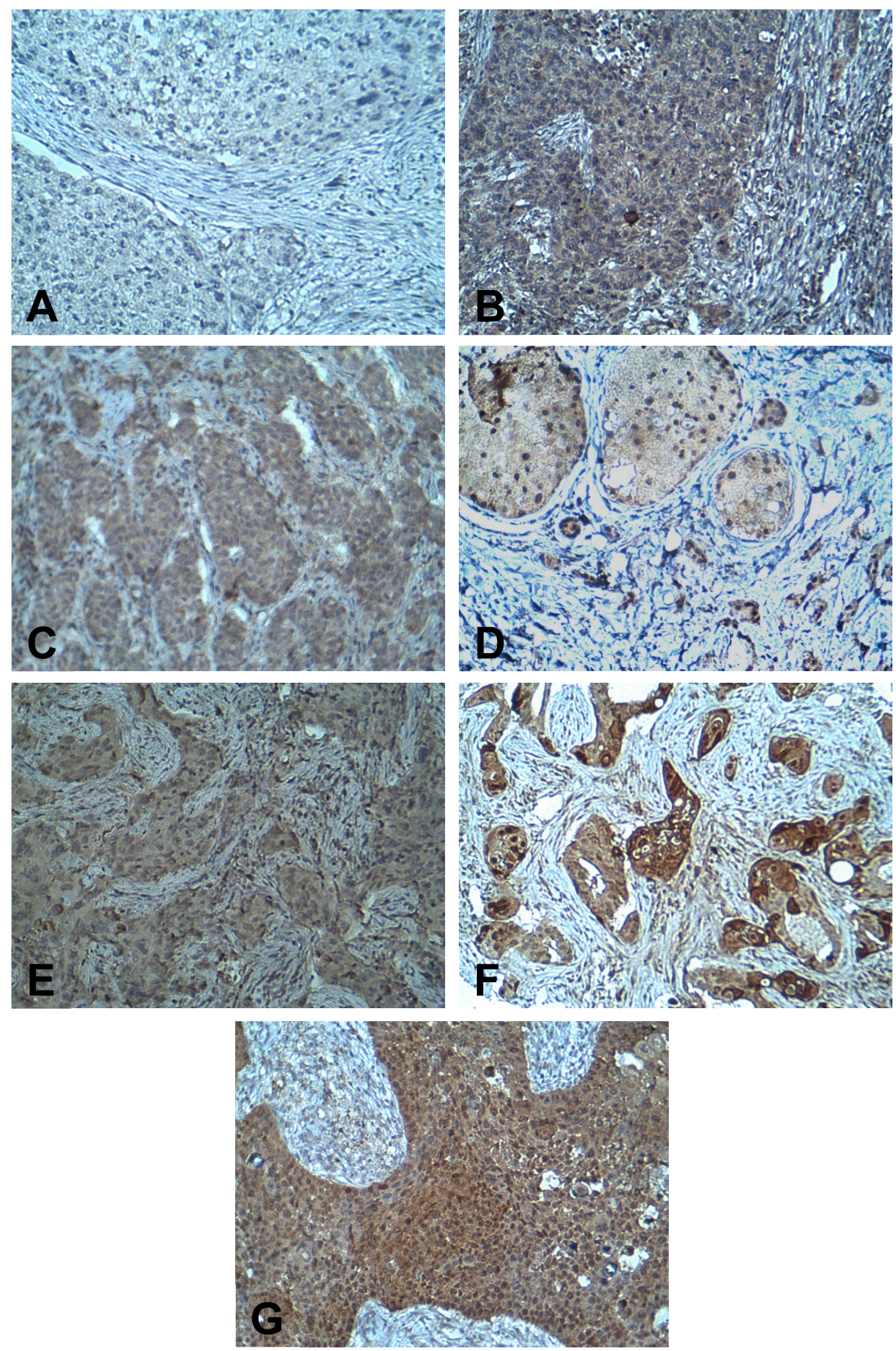

Figure 1. Immunohistochemistry staining for IR and c-MET in breast cancer. (A) Negative staining for IR; (B) mild staining for IR; (C) moderate staining for IR; (D) strongly positive staining for IR; (E) mild staining for c-MET; (F) moderate staining for c-MET; and (G) strongly positive staining for c-MET. [Magnification at x200]. IR, insulin receptor. 


\subsection{Statistical Analysis}

Data analysis was performed using IBM SPSS statistical package (IBM Corp. Version 21.0. Armonk, NY, USA). Continuous variables are expressed as mean \pm standard deviation or median and interquartile range (IQR). Categorical variables are presented as frequency and percentages (n, \%). A non-parametric Mann-Whitney $U$ test was applied to compare two independent groups. Kruskal-Wallis analysis of variance was used to compare multiple independent groups. To compare categorical variables between groups, Pearson's $\chi^{2}$ test of independence was used. To assess correlations between continuous variables, Spearman's correlation test was applied. All $p$-values were two-sided and statistical significance is indicated at $p<0.05$.

Dichotomization of some categorical variables was considered for correlation analysis of some study variables. This dichotomization was based on sample size and was performed in advance of conducting statistical analysis in order to avoid small sample size upon further stratification of data by menopausal status [28]. Therefore, BMI was categorized as non-obese $\left(<30.00 \mathrm{~kg} / \mathrm{m}^{2}\right)$ and obese $\left(\geq 30.0 \mathrm{~kg} / \mathrm{m}^{2}\right)$. The TNM stage of breast cancer was dichotomized as early (I/II) and advanced (III/IV). Histologic grade was further categorized into grades (I/II) and grade (III) tumors. Categories of these tumor variables were selected based on cut points previously published by other researchers [32,33]. Receptor expression was further categorized as either low or high based on IRS scoring. IRS scores of 7-12 were considered high, and scores below 7 were considered low for expression of target receptors [34,35].

Kaplan-Meier survival curves were generated for breast cancer patients based on the expression status of IR and c-MET using GraphPad Prism 8.0.1 software (GraphPad Software, San Diego, CA, USA). Cox proportional hazards models were fitted with overall survival (OS) as the outcome.

\section{Results}

\subsection{Patient Characteristics}

Demographic and clinicopathologic features of breast cancer patients are presented in Table 1. Mean age at presentation was $49.5 \pm 10.4$ ranging from $28-77$ years (median 47, IQR 42-57). Mean BMI was $29.7 \pm 5.3 \mathrm{~kg} / \mathrm{m}^{2}$ (range 18.7-41.2, median 29.1, IQR 25.8-33.3) and most patients (42.3\%) were obese. Thirty-seven patients $(52.1 \%)$ were premenopausal and 30 patients $(42.3 \%)$ were postmenopausal. Mean tumor size was $4.5 \pm 0.3 \mathrm{~cm}$ with a rage of $0.5-13$ (median 4, IQR 2.5-5.9). More than half of patients $(54.9 \%)$ had lymph node positive disease at presentation. Thirty patients presented at stage II disease (42.3\%) while 24 patients had stage III disease (33.8\%). Most patients $(70.4 \%)$ had grade III disease and invasive ductal carcinoma (IDC) was the most common histologic subtype $(81.7 \%)$. Twenty-nine patients $(40.8 \%)$ presented with triple-negative molecular subtype while (32.4\%) presented with luminal disease. Other clinicopathologic parameters are shown in Table 1.

Table 1. Demographics and clinicopathologic characteristics of breast cancer patients $(n=71)$.

\begin{tabular}{cc}
\hline Characteristics & n (\%) \\
\hline \multicolumn{1}{c}{ Age, years } & \\
$<45$ & $26(36.6)$ \\
$45-60$ & $33(46.5)$ \\
$>60$ & $12(16.9)$ \\
BMI, kg/m ${ }^{2}$ & \\
Normal & $16(22.5)$ \\
Overweight & $25(35.2)$ \\
Obese & $30(42.3)$ \\
Marital status & \\
Single & $8(11.3)$ \\
Married & $63(88.7)$ \\
\hline
\end{tabular}


Table 1. Cont.

\begin{tabular}{|c|c|}
\hline Characteristics & n (\%) \\
\hline \multicolumn{2}{|c|}{ Menopausal status } \\
\hline Premenopausal & $37(52.1)$ \\
\hline Postmenopausal & $30(42.3)$ \\
\hline Unknown & $4(5.6)$ \\
\hline \multicolumn{2}{|c|}{ Family history of breast cancer } \\
\hline Absent & $49(69.0)$ \\
\hline Present & $14(19.7)$ \\
\hline Unknown & $8(11.3)$ \\
\hline \multicolumn{2}{|c|}{ Smoking } \\
\hline Never smoker & $61(85.9)$ \\
\hline Past smoker & $3(4.2)$ \\
\hline Current smoker & $6(8.5)$ \\
\hline Unknown & $1(1.4)$ \\
\hline \multicolumn{2}{|c|}{ Alcohol intake } \\
\hline Never & $66(93.0)$ \\
\hline Unknown & $5(7.0)$ \\
\hline \multicolumn{2}{|c|}{ HRT use } \\
\hline No & $60(84.5)$ \\
\hline Yes & $1(1.4)$ \\
\hline Unknown & $10(14.1)$ \\
\hline \multicolumn{2}{|c|}{ Site of disease } \\
\hline Right & $35(49.3)$ \\
\hline Left & $33(46.5)$ \\
\hline Bilateral & $3(4.2)$ \\
\hline \multicolumn{2}{|l|}{ Tumor size } \\
\hline$<2 \mathrm{~cm}$ & $6(8.5)$ \\
\hline$\geq 2 \mathrm{~cm}$ & $59(83.1)$ \\
\hline Unknown & $6(8.5)$ \\
\hline \multicolumn{2}{|l|}{ Lymph nodes } \\
\hline Negative & $24(33.8)$ \\
\hline Positive & $39(54.9)$ \\
\hline Unknown & $8(11.3)$ \\
\hline \multicolumn{2}{|l|}{ Stage } \\
\hline I & $3(4.2)$ \\
\hline II & $30(42.3)$ \\
\hline III & $24(33.8)$ \\
\hline IV & $11(15.5)$ \\
\hline Unknown & $3(4.2)$ \\
\hline \multicolumn{2}{|c|}{ Grade } \\
\hline I & $5(7.0)$ \\
\hline II & $14(19.7)$ \\
\hline III & $50(70.4)$ \\
\hline Unknown & $2(2.8)$ \\
\hline \multicolumn{2}{|c|}{ Histologic type } \\
\hline IDC & $58(81.7)$ \\
\hline ILC & $11(15.5)$ \\
\hline Other & $2(2.8)$ \\
\hline \multicolumn{2}{|c|}{ LVI } \\
\hline Unidentified & $28(39.4)$ \\
\hline Identified & $37(52.1)$ \\
\hline Unknown & $6(8.5)$ \\
\hline \multicolumn{2}{|l|}{ ER } \\
\hline Positive & $23(32.4)$ \\
\hline Negative & $48(67.6)$ \\
\hline \multicolumn{2}{|c|}{ PR } \\
\hline Positive & $19(26.8)$ \\
\hline Negative & $52(73.2)$ \\
\hline
\end{tabular}


Table 1. Cont.

\begin{tabular}{cc}
\hline Characteristics & n (\%) \\
\hline \multicolumn{1}{c}{ HER2 } & $19(26.8)$ \\
Positive & $52(73.2)$ \\
Negative & \\
Molecular subtype & $23(32.4)$ \\
Luminal & $19(26.8)$ \\
HER2-positive & $29(40.8)$ \\
Triple-negative & \\
Surgery & $60(84.5)$ \\
Mastectomy & $11(15.5)$ \\
Wide local excision & $59(83.1)$ \\
Adjuvant chemotherapy \\
Yes & $4(5.6)$ \\
No & $8(11.3)$ \\
Unknown
\end{tabular}

BMI, body mass index. ER, estrogen receptor. HRT, hormone replacement therapy. HER2, human epidermal growth factors receptor 2. IDC, invasive ductal carcinoma. ILC, invasive lobular carcinoma. LVI, lymphovascular invasion. $\mathrm{PR}$, progesterone receptor.

\subsection{Expression of IR and c-MET in Breast Cancer Tissues and Association with Clinicopathologic Characteristics}

IR immunoreactivity was detected in $68 / 71$ patients $(95.8 \%)$ by IHC. IR was detected in cell membranes (Figure 1A-D). Mean IRS score for IR expression was $4.5 \pm 0.4$ ranging from 0-12 (median 4.0, IQR 2.0-6.0). According to IRS scoring, 8 patients (11.8\%) classified to have negative expression of IR, 22 patients (32.4\%) had mild staining, 31 patients $(45.6 \%)$ had moderate staining, and 7 patients $(10.3 \%)$ showed strongly positive staining (Figure $1 \mathrm{~A}-\mathrm{D})$.

The association between clinicopathologic characteristics of breast cancer patients and expression of IR is shown in Table 2. Fifty-three patients (74.6\%) were determined to have low expression of IR. IR expression was significantly associated with tumor grade $(p=0.017)$. Among patients with high IR expression, $42.1 \%$ presented with low-to-intermediate grade (I/II) compared to $14.9 \%$ of patients who had high grade carcinoma and high IR expression. IR expression was also significantly associated with ER expression $(p=0.015)$. Low IR expression was mostly found among patients with an ER-negative status (86.7\%). Alternatively, high IR expression was associated with an ER-positive status (39.1\%). No significant associations were found between IR expression and patient age, BMI, menopausal status, tumor stage, lymph node involvement, PR, HER2, or LVI (Table 2).

Expression of c-MET was detected in all cases and was mainly observed as cytoplasmic staining of breast cancer cells (Figure 1E-G). c-MET expression was determined to be strongly positive in 15 (21.1\%) cases, moderate in $48(67.6 \%)$, and mild in $8(11.3 \%)$ patients (Figure 1E-G). Mean immunoreactive score (IRS) for c-MET expression was $7.8 \pm 0.3$ with a range from 2-12 (median 8.0, IQR 6.0-8.0).

The association between c-MET expression and clinicopathologic characteristics of patients is presented in Table 3. Low c-MET expression was found in 19 (26.8\%) patients, while 52 (73.2\%) patients had high expression. No significant associations were found between c-MET expression and all demographic and clinicopathologic parameters assessed among the entire sample of breast cancer patients (Table 3).

Correlation between receptor expression scores and clinicopathologic characteristics associated with outcomes in breast cancer patients is shown in Table 4. There was a significant positive correlation between IR and c-MET expression scores among the entire cohort (rho $=0.458, p<0.001$ ). There were no significant correlations between IR or c-MET scores and age at diagnosis, BMI, tumor size, or number of lymph nodes among breast cancer patients (Table 4). 
Table 2. Expression of IR (insulin receptor) and its association with clinicopathologic parameters in breast cancer patients $(n=68)$.

\begin{tabular}{|c|c|c|c|}
\hline \multirow[b]{2}{*}{ Parameter } & \multicolumn{2}{|c|}{ IR [n (\%)] } & \multirow{2}{*}{$p$-Value } \\
\hline & $\begin{array}{c}\text { Low } \\
(n=53)\end{array}$ & $\begin{array}{c}\text { High } \\
(n=15)\end{array}$ & \\
\hline Age, years & & & 0.341 \\
\hline$<50$ & $28(73.7 \%)$ & $10(26.3 \%)$ & \\
\hline$\geq 50$ & $25(83.3 \%)$ & $5(16.7 \%)$ & \\
\hline Menopausal status & & & 0.195 \\
\hline Premenopausal & $26(72.2 \%)$ & $10(27.8 \%)$ & \\
\hline Postmenopausal & $24(85.7 \%)$ & $4(14.3 \%)$ & \\
\hline BMI, $\mathrm{kg} / \mathrm{m}^{2}$ & & & 0.533 \\
\hline Non-obese & $33(80.5 \%)$ & $8(19.5 \%)$ & \\
\hline Obese & $20(74.1 \%)$ & $7(25.9 \%)$ & \\
\hline Grade & & & 0.017 * \\
\hline $\mathrm{I} / \mathrm{II}$ & $11(57.9 \%)$ & $8(42.1 \%)$ & \\
\hline III & $40(85.1 \%)$ & $7(14.9 \%)$ & \\
\hline Stage & & & 0.635 \\
\hline Early (I and II) & $26(81.3 \%)$ & $6(18.8 \%)$ & \\
\hline Advanced (III and IV) & $26(76.5 \%)$ & $8(23.5 \%)$ & \\
\hline Lymph nodes & & & 0.571 \\
\hline Negative & $18(75.0 \%)$ & $6(25.0 \%)$ & \\
\hline Positive & $30(81.1 \%)$ & $7(18.9 \%)$ & \\
\hline ER & & & $0.015^{*}$ \\
\hline Positive & $14(60.9 \%)$ & $9(39.1 \%)$ & \\
\hline Negative & $39(86.7 \%)$ & $6(13.3 \%)$ & \\
\hline PR & & & 0.067 \\
\hline Positive & $12(63.2 \%)$ & $7(36.8 \%)$ & \\
\hline Negative & $41(83.7 \%)$ & $8(16.3 \%)$ & \\
\hline HER2 & & & 0.081 \\
\hline Positive & $15(93.8 \%)$ & $1(6.3 \%)$ & \\
\hline Negative & $39(73.1 \%)$ & $14(26.9 \%)$ & \\
\hline LVI & & & 0.244 \\
\hline Unidentified & $19(70.4 \%)$ & $8(29.6 \%)$ & \\
\hline Identified & $29(82.9 \%)$ & $6(17.1 \%)$ & \\
\hline
\end{tabular}

Low, IRS $=0-\overline{6, \text { high, IRS }=7-12 . \text { Pearson's chi-square test of independence. Statistically significant at }}{ }^{*} p<0.05$ BMI, body mass index. ER, estrogen receptor. HER2, human epidermal growth factor receptor 2. IR, insulin receptor. LVI, lymphovascular invasion. PR, progesterone receptor.

Table 3. Expression of c-MET and its association with clinicopathologic parameters in breast cancer patients $(n=71)$.

\begin{tabular}{cccc}
\hline & \multicolumn{2}{c}{ c-MET [n (\%)] } & \multirow{2}{*}{ Parameter } \\
\cline { 2 - 3 } & $\begin{array}{c}\text { Low } \\
(\mathbf{n}=\mathbf{1 9 )}\end{array}$ & $\begin{array}{c}\text { High } \\
(\mathbf{n}=\mathbf{5 2})\end{array}$ & \\
\hline Age, years & & & 0.064 \\
$<50$ & $7(17.9 \%)$ & $32(82.1 \%)$ & \\
$\geq 50$ & $12(37.5 \%)$ & $20(62.5 \%)$ & \multirow{2}{*}{0.057} \\
Menopausal status & $7(18.9 \%)$ & $30(81.1 \%)$ & \\
Premenopausal & $12(40.0 \%)$ & $18(60.0 \%)$ & \multirow{2}{*}{0.271} \\
Postmenopausal & & & \\
Body mass index (BMI), $\mathrm{kg} / \mathrm{m}^{2}$ & $13(31.7 \%)$ & $28(68.3 \%)$ & \\
Non-obese & $6(20.0 \%)$ & $24(80.0 \%)$ & \\
Obese & & & \\
\hline
\end{tabular}


Table 3. Cont

\begin{tabular}{|c|c|c|c|}
\hline \multirow[b]{2}{*}{ Parameter } & \multicolumn{2}{|c|}{ c-MET [n (\%)] } & \multirow{2}{*}{$p$-Value } \\
\hline & $\begin{array}{c}\text { Low } \\
(n=19)\end{array}$ & $\begin{array}{c}\text { High } \\
(n=52)\end{array}$ & \\
\hline Grade & & & 0.979 \\
\hline $\mathrm{I} / \mathrm{II}$ & $5(26.3 \%)$ & $14(73.7 \%)$ & \\
\hline III & $13(26.0 \%)$ & $37(74.0 \%)$ & \\
\hline Stage & & & 0.327 \\
\hline Early (I and II) & $10(30.3 \%)$ & $23(69.7 \%)$ & \\
\hline Advanced (III and IV) & $7(20.0 \%)$ & $28(80.0 \%)$ & \\
\hline Lymph nodes & & & 0.373 \\
\hline Negative & $8(33.3 \%)$ & $16(66.7 \%)$ & \\
\hline Positive & $9(23.1 \%)$ & $30(76.9 \%)$ & \\
\hline ER & & & 0.929 \\
\hline Positive & $6(26.1 \%)$ & $17(73.9 \%)$ & \\
\hline Negative & $13(27.1 \%)$ & $35(72.9 \%)$ & \\
\hline PR & & & 0.959 \\
\hline Positive & $5(26.3 \%)$ & $14(73.7 \%)$ & \\
\hline Negative & $14(26.9 \%)$ & $38(73.1 \%)$ & \\
\hline HER2 & & & 0.511 \\
\hline Positive & $4(21.1 \%)$ & $15(78.9 \%)$ & \\
\hline Negative & $15(28.8 \%)$ & $37(71.2 \%)$ & \\
\hline LVI & & & 0.700 \\
\hline Unidentified & $8(28.6 \%)$ & $20(71.4 \%)$ & \\
\hline Identified & $9(24.3 \%)$ & $28(75.7 \%)$ & \\
\hline
\end{tabular}

Low, IRS $=0-6$, high, IRS $=7-12$. Pearson's chi-square test of independence. Statistically significant at * $p<0.05$. BMI, body mass index. ER, estrogen receptor. HER2, human epidermal growth factor receptor 2. LVI, lymphovascular invasion. PR, progesterone receptor.

Table 4. Correlation between IR and c-MET expression with demographic and clinicopathologic factors in breast cancer patients (Continuous variables) $(\mathrm{n}=71)$.

\begin{tabular}{ccccc}
\hline \multirow{2}{*}{ Parameter } & \multicolumn{2}{c}{ IR Score } & \multicolumn{2}{c}{ c-MET Score } \\
\cline { 2 - 5 } & rho & $\boldsymbol{p}$-Value & rho & $\boldsymbol{p}$-Value \\
\hline Age, years & 0.054 & 0.659 & -0.103 & 0.394 \\
BMI, kg/m & 0.155 & 0.207 & 0.001 & 0.991 \\
Tumor size, cm & 0.029 & 0.823 & 0.129 & 0.306 \\
Lymph nodes & 0.199 & 0.130 & 0.151 & 0.244 \\
IR score & - & - & $\mathbf{0 . 4 5 8}$ & $<0.001$ * \\
c-MET score & 0.458 & $<0.001 *$ & -
\end{tabular}

rho, Spearman's correlation coefficient. Statistically significant at ${ }^{*} p<0.05$. BMI, body mass index. IR, insulin receptor.

\subsection{Correlation between IR and c-MET Expression with Clinicopathologic Characteristics Based on Menopausal Status}

Correlations between IR and c-MET scores and clinicopathologic factors associated with outcomes in breast cancer patients stratified according to menopausal status are shown in Tables 5 and 6 , respectively. Among premenopausal cases, IR expression scores were significantly higher in patients with grade I/II disease $(p=0.025)$, ER-positive $(p=0.030)$, and PR-positive carcinoma $(p=0.015)$ (Table 5). There were no significant correlations between IR scores with tumor stage, lymph node status, HER2, and LVI among premenopausal breast cancer patients. Among postmenopausal cases, IR immunostaining scores lacked significant correlation with all clinicopathologic variables tested.

c-MET expression scores were significantly higher among premenopausal patients with ER-positive $(p=0.007)$ and PR-positive carcinoma $(p=0.024)$ (Table 6). There were no significant correlations between c-MET scores with the other tumor characteristics among premenopausal cases. Similar to 
findings with IR, c-MET expression lacked significant correlations with all clinicopathologic variables tested among postmenopausal breast cancer patients in this study (Table 6).

Table 5. Correlation between IR expression with clinicopathologic factors stratified by menopausal status among breast cancer patients $(n=67)$.

\begin{tabular}{|c|c|c|c|c|}
\hline \multirow{3}{*}{ Parameter } & \multicolumn{2}{|c|}{$\begin{array}{l}\text { Premenopausal } \\
(\mathrm{n}=37)\end{array}$} & \multicolumn{2}{|c|}{$\begin{array}{l}\text { Postmenopausal } \\
\quad(n=30)\end{array}$} \\
\hline & IR Score & V V & IR Score & Vlulu \\
\hline & Median (IQR) & & Median (IQR) & \\
\hline Grade & & $0.025 *$ & & 0.978 \\
\hline $\mathrm{I} / \mathrm{II}$ & $8.0(4.0-12.0)$ & & $3.0(2.0-8.0)$ & \\
\hline III & $4.0(2.0-6.0)$ & & $3.5(3.0-4.0)$ & \\
\hline Stage & & 0.686 & & 0.085 \\
\hline Early (I/II) & $4.0(2.0-8.0)$ & & $3.0(1.0-4.0)$ & \\
\hline Advanced (III/IV) & $4.0(3.0-7.5)$ & & $3.5(3.0-5.0)$ & \\
\hline Lymph nodes & & 0.944 & & 0.085 \\
\hline Negative & $4.0(2.0-8.0)$ & & $2.5(2.0-3.75)$ & \\
\hline Positive & $4.0(3.0-6.5)$ & & $3.5(3.0-4.0)$ & \\
\hline ER & & 0.030 * & & 0.376 \\
\hline Positive & $4.0(4.0-12.0)$ & & $3.5(2.75-8.0)$ & \\
\hline Negative & $4.0(2.0-6.0)$ & & $3.0(2.0-4.00)$ & \\
\hline PR & & 0.015 * & & 0.404 \\
\hline Positive & $6.0(4.0-12.0)$ & & $3.5(3.0-7.0)$ & \\
\hline Negative & $3.5(2.0-6.0)$ & & $3.0(2.0-4.0)$ & \\
\hline HER2 & & 0.429 & & 0.198 \\
\hline Positive & $6.0(2.5-9.0)$ & & $4.0(3.0-4.0)$ & \\
\hline Negative & $4.0(2.0-8.0)$ & & $3.0(2.0-4.0)$ & \\
\hline LVI & & 0.986 & & 0.834 \\
\hline Unidentified & $4.0(1.0-12.0)$ & & $3.0(2.0-4.0)$ & \\
\hline Identified & $4.0(3.0-6.0)$ & & $3.0(3.0-4.0)$ & \\
\hline
\end{tabular}

Mann-Whitney U test. Statistically significant at ${ }^{*} p<0.05$. ER, estrogen receptor. HER2, human epidermal growth factor receptor 2. IR, insulin receptor. IQR, interquartile range. LVI, lymphovascular invasion. PR, progesterone receptor.

Table 6. Correlation between c-MET expression with clinicopathologic factors stratified by menopausal status among breast cancer patients $(n=67)$.

\begin{tabular}{|c|c|c|c|c|}
\hline \multirow{3}{*}{ Parameter } & \multicolumn{2}{|c|}{$\begin{array}{l}\text { Premenopausal } \\
(\mathrm{n}=37)\end{array}$} & \multicolumn{2}{|c|}{$\begin{array}{l}\text { Postmenopausal } \\
\qquad(\mathrm{n}=30)\end{array}$} \\
\hline & c-MET Score & VIn & c-MET Score & VOlu \\
\hline & Median (IQR) & & Median (IQR) & \\
\hline Grade & & 0.404 & & 0.780 \\
\hline I/II & $8.0(8.0-12.0)$ & & $7.0(3.75-9.0)$ & \\
\hline III & $8.0(7.5-12.0)$ & & $8.0(3.75-8.0)$ & \\
\hline Stage & & 0.911 & & 0.143 \\
\hline Early (I/II) & $8.0(8.0-12.0)$ & & $7.0(3.0-8.0)$ & \\
\hline Advanced (III/IV) & $8.0(8.0-11.0)$ & & $8.0(6.0-8.0)$ & \\
\hline Lymph nodes & & 0.569 & & 0.143 \\
\hline Negative & $8.0(8.0-12.0)$ & & $8.0(5.0-8.0)$ & \\
\hline Positive & $8.0(8.0-9.0)$ & & $5.0(3.0-8.0)$ & \\
\hline ER & & $0.007 *$ & & 0.317 \\
\hline Positive & $12.0(8.0-12.0)$ & & $5.0(3.0-9.0)$ & \\
\hline Negative & $8.0(6.0-8.0)$ & & $8.0(6.0-8.0)$ & \\
\hline PR & & 0.024 * & & 0.372 \\
\hline Positive & $10.0(8.0-12.0)$ & & $5.0(3.0-11.0)$ & \\
\hline Negative & $8.0(6.0-8.0)$ & & $8.0(5.5-8.0)$ & \\
\hline
\end{tabular}


Table 6. Cont.

\begin{tabular}{|c|c|c|c|c|}
\hline \multirow{3}{*}{ Parameter } & \multicolumn{2}{|c|}{$\begin{array}{l}\text { Premenopausal } \\
\qquad(n=37)\end{array}$} & \multicolumn{2}{|c|}{$\begin{array}{l}\text { Postmenopausal } \\
\qquad(\mathrm{n}=30)\end{array}$} \\
\hline & c-MET Score & n-Value & c-MET Score & $n$-Value \\
\hline & Median (IQR) & & Median (IQR) & \\
\hline HER2 & & 0.117 & & 0.083 \\
\hline Positive & $7.0(3.75-9.0)$ & & $8.0(8.0-8.0)$ & \\
\hline Negative & $8.0(8.0-12.0)$ & & $6.0(3.0-8.0)$ & \\
\hline LVI & & 0.956 & & 0.238 \\
\hline Unidentified & $8.0(8.0-11.0)$ & & $8.0(3.0-8.0)$ & \\
\hline Identified & $8.0(8.0-12.0)$ & & $8.0(4.0-8.0)$ & \\
\hline
\end{tabular}

Mann-Whitney U test. Statistically significant at ${ }^{*} p<0.05$ ER, estrogen receptor. HER2, human epidermal growth factor receptor 2. IR, insulin receptor. IQR, interquartile range. LVI, lymphovascular invasion. PR, progesterone receptor.

\subsection{Expression of IR and c-MET among Breast Cancer Molecular Subtypes}

Figure 2 represents IR and c-MET immunostaining scores among breast cancer molecular subtypes for all patients including premenopausal and postmenopausal cases. IR score was significantly different among molecular subtypes for all patients $(p=0.006)$ and premenopausal cases $(p=0.035)$ (Figure 2A,B). IR scores were significantly higher among patients with luminal subtype compared to both HER2-positive and triple-negative disease. No significant difference in IR score was found among molecular subtypes in postmenopausal patients (Figure 2C). Expression of c-MET was statistically different among molecular subtypes for premenopausal patients $(p=0.019)$ with higher expression scores for the luminal subtype compared with HER2-positive and triple-negative cases (Figure 2E).

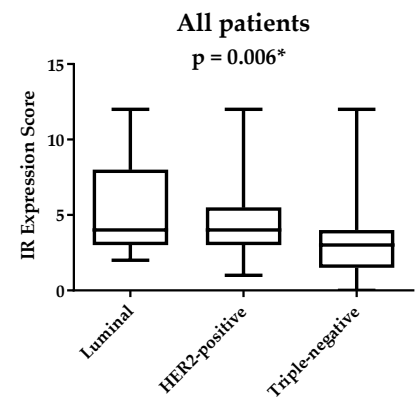

(A)

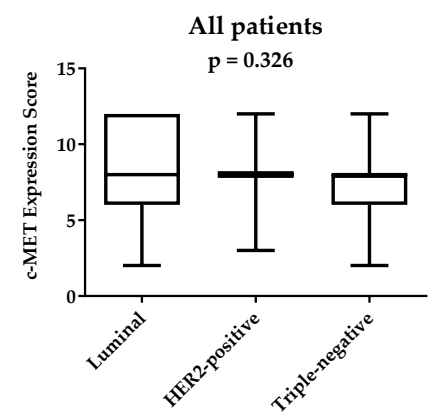

(D)

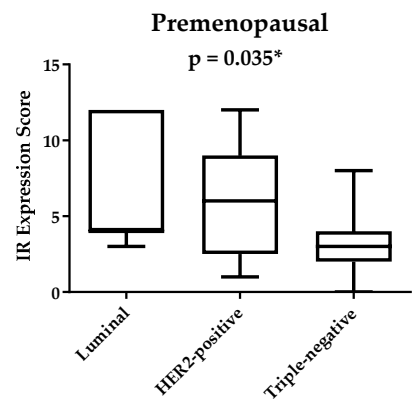

(B)

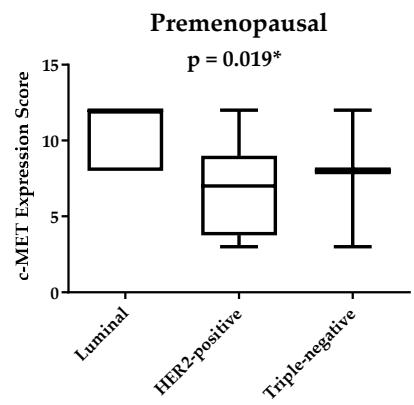

(E)

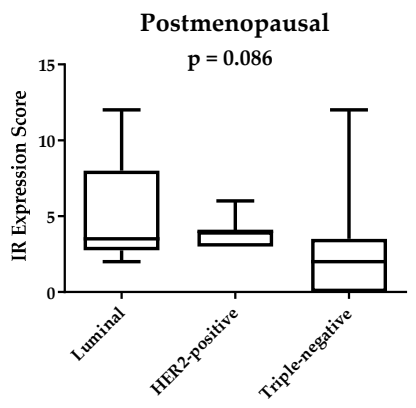

(C)

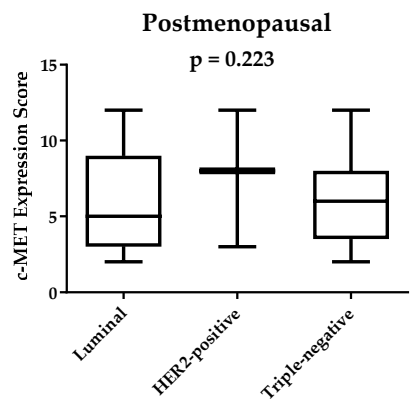

(F)

Figure 2. Expression of IR and c-MET among breast cancer patients according to molecular subtype and menopausal status. IR, insulin receptor. Boxplots represent median IR scores (A-C) and c-MET scores (D-F). The boxes represent the 25th and 75th percentiles, whereas the bars represent minimum and maximum values. ${ }^{*} p<0.05$ between molecular subtypes, according to Kruskal-Wallis analysis of variance. 


\subsection{Survival Analysis}

Figure 3 shows the overall survival (OS) of breast cancer patients stratified by the expression status of IR and c-MET. Survival curves for patients with low or high expression of IR were not significantly different ( $p=0.643$, Log-rank (Mantel-Cox) test). Similarly, the expression status of c-MET did not result in significant differences in patient OS ( $p=0.527$, Log-rank (Mantel-Cox) test).
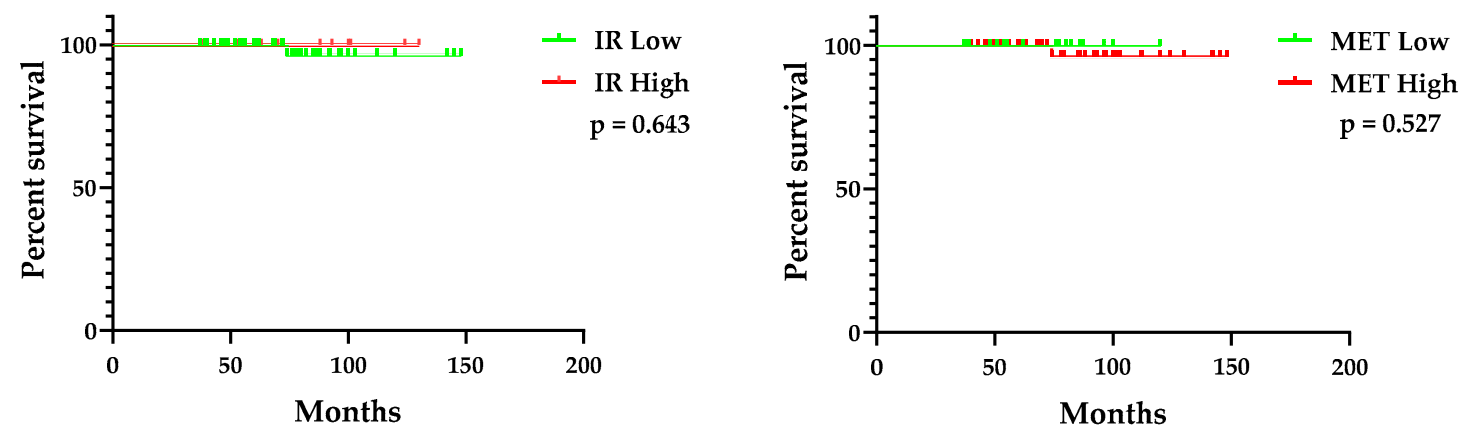

Figure 3. Overall survival of breast cancer patients by expression status of IR (left panel) and c-MET (right panel). IR, insulin receptor.

\section{Discussion}

Insulin is an established mitogen known to promote breast cancer cell growth [10,36]. The mitogenic actions of insulin in breast cancer cells are mediated by activation of IR. Earlier studies showed that IR was overexpressed in breast tumors when compared to normal breast tissue [10,37]. In cancer cells, IR has been found to drive proliferative and mitogenic effects, which are distinct from the regular metabolic activity mediated by the receptor [5,18]. Activation of IR phosphorylates IR substrate (IRS) which, in turn, activates multiple downstream signal transduction effectors such as phosphoinositide 3-kinase (PI3K)/AKT and ERK pathways [38,39]. As a result, IR regulates several fundamental cellular processes such as cell proliferation, differentiation, cell cycle, and migration [17]. Insulin-like growth factor 1 receptor (IGF-1R) is a common dimerization partner of IR [5,7]. IGF-1R/IR pathway mediated resistance to endocrine treatment and is further required for the growth of hormone-independent breast cancer $[7,38,40]$.

A limited number of studies described the expression of IR in breast cancer tissues and its association with disease presentation and prognosis [41]. Results from this study demonstrated that high IR expression was significantly associated with low tumor grade and expression of hormone receptors, which are classified as favorable prognostic factors [42]. When stratified by menopausal status, these associations were found among premenopausal but not postmenopausal patients. Our findings showed no significant associations between IR expression with patient age, BMI, tumor size, or nodal status. In addition, our findings showed that patient survival was not affected by the IR expression status. In agreement with our findings, Mulligan et al. found that high IR expression was significantly associated with low tumor grade, lymph node negativity, and positive expression of PR in a series of breast cancer patients who underwent surgical treatment at three University of Toronto hospitals [43]. High IR expression was associated with improved distant disease-free survival and OS among breast cancer patients compared to low expression status [43]. In addition, Björner et al. described the expression of IR in a population-based cohort of 1,026 primary invasive breast cancer patients in Sweden [38]. Moderate-strong staining of IR was detected in $82 \%$ of breast cancer patients [38]. Strong IR expression was significantly associated with ER-positivity, while negative-weak expression of IR was associated with a triple-negative molecular status. However, IR expression lacked prognostic impact regarding event-free survival or distant metastasis among this cohort of Sweden breast cancer patients [38]. Alternatively, Papa et al. examined the expression of IR in 159 specimens for Italian breast cancer patients and compared the expression to the content of 42 non-malignant breast tissues [10]. The expression of IR in breast cancer tissues was more than six-fold higher than 
its expression in non-malignant breast tissue. In addition, Papa et al. showed that IR expression was positively correlated with tumor size, histological grade, and ER content [10]. No correlations were found between IR levels and age, body weight, menopausal status, and nodal involvement of breast cancer patients [10]. Aljada et al. showed that mRNA levels of IR were significantly higher in cancer patients presented with advanced clinical stage of the disease compared to early stages [44]. As our findings demonstrate, a favorable prognostic impact for IR expression in breast cancer tissues and IR expression may be perceived as a marker of differentiation [43].

c-MET is a high-affinity receptor for HGF [45]. c-MET is classified as the prototype member of the RTK subfamily with Ron and Sea based on shared structural homology of c-MET [46]. HGF binding induces c-MET dimerization and subsequent activation of several downstream transducers and adaptors including PI3K, Src, Grb2, Shc, and signal transducer and activator of transcription (STATs) [45]. Dysregulation of c-MET signaling upregulates diverse tumor cell functions including cell proliferation, survival, cell scattering and motility, epithelial-to-mesenchymal transition, angiogenesis, invasion, and metastasis [47]. In addition, high c-MET expression is critical for acquiring resistance toward chemotherapy and targeted therapies [48,49]. Findings from this study revealed that c-MET is strongly expressed in breast cancer tissues and its levels are associated with hormone-receptor expression and luminal subtype among premenopausal breast cancer patients. However, no associations were found between c-MET expression and other tumor characteristics or patient survival in this analysis. In agreement with our results, several studies revealed a lack of association between c-MET expression and prognostic factors such as patient age, tumor size, and lymph node status [50]. Wang et al. demonstrated a lack of correlation between c-MET expression and clinicopathologic characteristics or prognosis among a cohort of patients diagnosed with triple-negative breast cancer [51]. Lengyel et al. evaluated the expression of c-MET in 40 primary breast cancer patients with positive axillary lymph nodes in Germany and revealed a lack of association between receptor levels and age, lymph node involvement, ER, PR, tumor size, or grade [52]. Nakopoulou et al. showed that c-MET immunoreactivity was associated with low histological grade and favorable prognostic and predictive factors such as ER and PR positivity and negative HER2 expression [53]. Nevertheless, c-MET expression was not significantly associated with other prognostic factors such as tumor size, lymph node status, and stage [53]. Baccelli et al. showed that expression of c-MET in luminal tumors was associated with lymph node metastasis and reduced OS suggesting that c-MET expression could be utilized as a prognostic marker for risk stratification and treatment of patients with hormone receptor-positive breast cancer [54]. Jia et al. also showed that increased c-MET expression in breast cancer tissues was associated with increased tumor size and stage, and reduced OS [48]. c-MET expression was significantly associated with the tumor grade in a series of patients diagnosed with triple-negative breast cancer from Greece and Austria [55]. Results from a meta-analysis of 6010 breast cancer cases indicated that c-MET overexpression correlated with poor relapse-free survival (RFS) and OS in Western patients. However, such an association was not observed among Asian patients [56]. Inconsistencies in findings correlating IR and c-MET expression with the clinicopathologic characteristics of breast cancer or the prognostic impact could be attributed to different approaches applied to assess immunostaining of target receptors and differences in populations examined.

Our findings revealed that breast cancer tissues showed a significant positive correlation for the expression of IR and c-MET. A limited number of studies addressed the potential crosstalk between IR and c-MET. Fafalios et al. reported that c-MET is essential for an optimal response to insulin in hepatocytes in which c-MET directly engage with IR in a hybrid complex of both receptors [24]. Though this finding has been shown in hepatic tissue, it is possible that IR and c-MET receptor hybrids are formed in cancerous tissues as well. This is particularly important since high expression of both receptors was found among luminal disease and, thus, mitogenic signaling in these tumors could be, in part, dependent on IR and c-MET RTKs, which could enable luminal tumors to escape the suppressive effects of endocrine treatments. 
Expression of IR and c-MET in breast cancer tissues could make them sensitive to inhibitors targeted for both receptors. Traditionally, IR pathway has been the focus of antidiabetic therapies [5]. IR had received less attention as a therapeutic target in cancer because of the potential impact on glucose homeostasis [7]. Nevertheless, recent evidence from in vitro and in vivo studies had indicated that inhibitors of IR have potent antitumor activity [17,57-60]. Sharma et al. showed that IR antagonist S961 suppressed growth and downregulated IR expression in breast cancer cells [17]. KW-2450 (Kyowa Kirin Pharmaceutical Development, Inc., Princeton, NJ, USA) is an orally active multi-targeted tyrosine kinase inhibitor with both IGF-1R and IR inhibitory activity [58]. KW-2450 exerts synergistic antitumor effects in combination with lapatinib and letrozole. This drug is underway in early phase clinical trials to evaluate tolerability and efficacy in combination with lapatinib plus letrozole in patients with HER2-positive advanced or metastatic breast cancer [58]. The dual IGF-1R/IR tyrosine kinase inhibitor BMS-754807 (Bristol-Myers Squibb, New York, NY, USA) resulted in synergistic antitumor effects in combination with letrozole or tamoxifen in preclinical breast cancer models [59]. OSI-906 (OSI Pharmaceuticals, Melville, NY, USA) is another dual tyrosine kinase inhibitor of IGF-1R/IR that has been shown to effectively suppress the growth of hormone-independent breast tumors in the animal model alone, and in combination with the ER down regulator fulvestrant [7]. Several c-MET inhibitors are commercially available, and some are approved for treatment of human cancers [47]. Currently, c-MET inhibitors are being investigated in breast cancer clinical trials as monotherapy or in combination with targeted treatments [61,62].

The main limitation of this study is the comparatively small sample size of our breast cancer patients. This may explain the lack of statistical significance for the association and correlation analysis performed in this study. However, patients in our study are included from a single center with unified diagnostic and management protocols. It is highly recommended to extend this analysis to involve a larger number of breast cancer patients to better elucidate the impact of receptor expression on clinicopathologic and prognostic factors.

\section{Conclusions}

To the best of our knowledge, this is the first study to characterize the expression of IR and c-MET in Jordanian breast cancer patients and describe associations with clinicopathologic and prognostic factors. Collectively, IR and c-MET were expressed in different molecular subtypes of breast cancer. The majority of patients had high tumor expression of c-MET. Expression of IR was associated with hormone receptors and tumor grade, which could imply a greater prognostic value for IR compared to c-MET. c-MET expression was associated with hormone receptors but lacked significant associations with other prognostic factors. When stratified based on menopausal status, these associations were confined to premenopausal cases. Despite their association with favorable prognostic factors in this study, ongoing research is being directed toward developing inhibitors of IR and c-MET, which could benefit a specific population of breast cancer patients. Future research will be needed to understand the potential crosstalk between IR and c-MET in breast cancer.

Author Contributions: Conceptualization, N.M.A. Methodology, N.M.A., A.H.A.-M., and N.A. Validation, A.H.A.-M. and N.A. Formal analysis, N.M.A., I.I.M., and M.E.E. Resources, R.J.Y., M.E.E., and I.I.M. Writing-original draft preparation, N.M.A. Writing-review and editing, N.M.A. and R.J.Y. Funding acquisition, N.M.A. All authors have read and agreed to the published version of the manuscript.

Funding: This research was funded by the Deanship of Research at Jordan University of Science and Technology (JUST), grant number 20150029.

Conflicts of Interest: The authors declare no conflict of interest.

\section{References}

1. Hynes, N.E.; Watson, C.J. Mammary gland growth factors: Roles in normal development and in cancer. Cold Spring Harb. Perspect. Biol. 2010, 2, a003186. [CrossRef] [PubMed] 
2. Robinson, D.R.; Wu, Y.M.; Lin, S.F. The protein tyrosine kinase family of the human genome. Oncogene 2000, 19, 5548-5557. [CrossRef] [PubMed]

3. Sangwan, V.; Park, M. Receptor tyrosine kinases: Role in cancer progression. Curr. Oncol. 2006, 13, 191-193. [PubMed]

4. Favoni, R.E.; de Cupis, A. The role of polypeptide growth factors in human carcinomas: New targets for a novel pharmacological approach. Pharmacol. Rev. 2000, 52, 179-206.

5. Malaguarnera, R.; Belfiore, A. The insulin receptor: A new target for cancer therapy. Front. Endocrinol. 2011, 2, 93. [CrossRef]

6. Clayton, p.E.; Banerjee, I.; Murray, P.G.; Renehan, A.G. Growth hormone, the insulin-like growth factor axis, insulin and cancer risk. Nat. Rev. Endocrinol. 2011, 7, 11-24. [CrossRef]

7. Fox, E.M.; Miller, T.W.; Balko, J.M.; Kuba, M.G.; Sanchez, V.; Smith, R.A.; Liu, S.; Gonzalez-Angulo, A.M.; Mills, G.B.; Ye, F.; et al. A kinome-wide screen identifies the insulin/IGF-I receptor pathway as a mechanism of escape from hormone dependence in breast cancer. Cancer Res. 2011, 71, 6773-6784. [CrossRef]

8. Belfiore, A.; Frasca, F. IGF and insulin receptor signaling in breast cancer. J. Mammary Gland. Biol. Neoplasia. 2008, 13, 381-406. [CrossRef]

9. Kalla Singh, S.; Brito, C.; Tan, Q.W.; De Leon, M.; De Leon, D. Differential expression and signaling activation of insulin receptor isoforms A and B: A link between breast cancer and diabetes. Growth Factors 2011, 29, 278-289. [CrossRef]

10. Papa, V.; Pezzino, V.; Costantino, A.; Belfiore, A.; Giuffrida, D.; Frittitta, L.; Vannelli, G.B.; Brand, R.; Goldfine, I.D.; Vigneri, R. Elevated insulin receptor content in human breast cancer. J. Clin. Investig. 1990, 86, 1503-1510. [CrossRef]

11. Ligibel, J.A.; Strickler, H.D. Obesity and its impact on breast cancer: Tumor incidence, recurrence, survival, and possible interventions. Am. Soc. Clin. Oncol. Educ. Book. 2013, 33, 52-59. [CrossRef] [PubMed]

12. Novosyadlyy, R.; Lann, D.E.; Vijayakumar, A.; Rowzee, A.; Lazzarino, D.A.; Fierz, Y.; Carboni, J.M.; Gottardis, M.M.; Pennisi, P.A.; Molinolo, A.A.; et al. Insulin-mediated acceleration of breast cancer development and progression in a nonobese model of type 2 diabetes. Cancer Res. 2010, 70, 741-751. [CrossRef] [PubMed]

13. Kaaks, R.; Lukanova, A. Effects of weight control and physical activity in cancer prevention: Role of endogenous hormone metabolism. Ann. N. Y. Acad. Sci. 2002, 963, 268-281. [CrossRef] [PubMed]

14. Strickler, H.D.; Wylie-Rosett, J.; Rohan, T.; Hoover, D.R.; Smoller, S.; Burk, R.D.; Yu, H. The relation of type 2 diabetes and cancer. Diabetes Technol. Ther. 2001,3, 263-274. [CrossRef]

15. Vigneri, P.; Frasca, F.; Sciacca, L.; Pandini, G.; Vigneri, R. Diabetes and cancer. Endocr. Relat. Cancer 2009, 16, 1103-1123. [CrossRef]

16. Vigneri, P.; Frasca, F.; Sciacca, L.; Frittitta, L.; Vigneri, R. Obesity and cancer. Nutr. Metab. Cardiovasc. Dis. 2006, 16, 1-7. [CrossRef]

17. Sharma, P.; Kumar, S. S961, a biosynthetic insulin receptor antagonist, downregulates insulin receptor expression \& suppresses the growth of breast cancer cells. Indian J. Med Res. 2018, 147, 545-551. [CrossRef]

18. Dowling, R.J.; Niraula, S.; Chang, M.C.; Done, S.J.; Ennis, M.; McCready, D.R.; Leong, W.L.; Escallon, J.M.; Reedijk, M.; Goodwin, P.J.; et al. Changes in insulin receptor signaling underlie neoadjuvant metformin administration in breast cancer: A prospective window of opportunity neoadjuvant study. Breast Cancer Res. 2015, 17, 32. [CrossRef]

19. Gastaldi, S.; Comoglio, P.M.; Trusolino, L. The Met oncogene and basal-like breast cancer: Another culprit to watch out for? Breast Cancer Res. 2010, 12, 208. [CrossRef]

20. Sattler, M.; Salgia, R. c-Met and hepatocyte growth factor: Potential as novel targets in cancer therapy. Curr. Oncol. Rep. 2007, 9, 102-108. [CrossRef]

21. Gonzalez-Angulo, A.M.; Chen, H.; Karuturi, M.S.; Chavez-MacGregor, M.; Tsavachidis, S.; Meric-Bernstam, F.; Do, K.A.; Hortobagyi, G.N.; Thompson, P.A.; Mills, G.B.; et al. Frequency of mesenchymal-epithelial transition factor gene (MET) and the catalytic subunit of phosphoinositide-3-kinase (PIK3CA) copy number elevation and correlation with outcome in patients with early stage breast cancer. Cancer 2013, 119, 7-15. [CrossRef] [PubMed] 
22. Ponzo, M.G.; Lesurf, R.; Petkiewicz, S.; O’Malley, F.P.; Pinnaduwage, D.; Andrulis, I.L.; Bull, S.B.; Chughtai, N.; Zuo, D.; Souleimanova, M.; et al. Met induces mammary tumors with diverse histologies and is associated with poor outcome and human basal breast cancer. Proc. Natl. Acad. Sci. USA 2009, 106, 12903-12908. [CrossRef] [PubMed]

23. Ho-Yen, C.M.; Green, A.R.; Rakha, E.A.; Brentnall, A.R.; Ellis, I.O.; Kermorgant, S.; Jones, J.L. C-Met in invasive breast cancer: Is there a relationship with the basal-like subtype? Cancer 2014, 120, $163-171$. [CrossRef] [PubMed]

24. Fafalios, A.; Ma, J.; Tan, X.; Stoops, J.; Luo, J.; Defrances, M.C.; Zarnegar, R. A hepatocyte growth factor receptor (Met)-insulin receptor hybrid governs hepatic glucose metabolism. Nat. Med. 2011, 17, 1577-1584. [CrossRef]

25. World Health Organization (WHO). Obesity: Preventing and Managing the Global Epidemic; Technical Report for a WHO Consulation (WHO Technical Report Series 894); WHO: Geneva, Switzerland, 2000.

26. Edge, S.; Byrd, D.R.; Compton, C.C.; Fritz, A.G.; Greene, F.; Trotti, A. AJCC Cancer Staging Handbook from the AJCC Cancer Stagnig Manual, 7th ed.; Springer: New York, NY, USA, 2010.

27. Rakha, E.A.; Reis-Filho, J.S.; Baehner, F.; Dabbs, D.J.; Decker, T.; Eusebi, V.; Fox, S.B.; Ichihara, S.; Jacquemier, J.; Lakhani, S.R.; et al. Breast cancer prognostic classification in the molecular era: The role of histological grade. Breast Cancer Res. 2010, 12, 207. [CrossRef]

28. Stark, A.; Stahl, M.S.; Kirchner, H.L.; Krum, S.; Prichard, J.; Evans, J. Body mass index at the time of diagnosis and the risk of advanced stages and poorly differentiated cancers of the breast: Findings from a case-series study. Int. J. Obes. 2010, 34, 1381-1386. [CrossRef]

29. Spitale, A.; Mazzola, P.; Soldini, D.; Mazzucchelli, L.; Bordoni, A. Breast cancer classification according to immunohistochemical markers: Clinicopathologic features and short-term survival analysis in a population-based study from the South of Switzerland. Ann. Oncol. 2009, 20, 628-635. [CrossRef]

30. Remmele, W.; Stegner, H.E. Recommendation for uniform definition of an immunoreactive score (IRS) for immunohistochemical estrogen receptor detection (ER-ICA) in breast cancer tissue. Pathologe 1987, 8, 138-140.

31. Fedchenko, N.; Reifenrath, J. Different approaches for interpretation and reporting of immunohistochemistry analysis results in the bone tissue-A review. Diagn. Pathol. 2014, 9, 221. [CrossRef]

32. Ademuyiwa, F.O.; Groman, A.; O'Connor, T.; Ambrosone, C.; Watroba, N.; Edge, S.B. Impact of body mass index on clinical outcomes in triple-negative breast cancer. Cancer 2011, 117, 4132-4140. [CrossRef]

33. Vona-Davis, L.; Rose, D.P.; Hazard, H.; Howard-McNatt, M.; Adkins, F.; Partin, J.; Hobbs, G. Triple-negative breast cancer and obesity in a rural Appalachian population. Cancer Epidemiol. Biomark. Prev. 2008, 17, 3319-3324. [CrossRef] [PubMed]

34. Li, Y.H.; Gao, H.F.; Wang, Y.; Liu, F.; Tian, X.F.; Zhang, Y. Overexpression of Gli1 in cancer interstitial tissues predicts early relapse after radical operation of breast cancer. Chinese J. Cancer Res. = Chung-kuo yen cheng yen chiu 2012, 24, 263-274. [CrossRef]

35. Ten Haaf, A.; Bektas, N.; von Serenyi, S.; Losen, I.; Arweiler, E.C.; Hartmann, A.; Knuchel, R.; Dahl, E. Expression of the glioma-associated oncogene homolog (GLI) 1 in human breast cancer is associated with unfavourable overall survival. BMC Cancer 2009, 9, 298. [CrossRef]

36. Dai, L.; Qi, Y.; Chen, J.; Kaczorowski, D.; Di, W.; Wang, W.; Xia, P. Sphingosine kinase (SphK) 1 and SphK2 play equivalent roles in mediating insulin's mitogenic action. Mol. Endocrinol. 2014, 28, 197-207. [CrossRef] [PubMed]

37. Sciacca, L.; Costantino, A.; Pandini, G.; Mineo, R.; Frasca, F.; Scalia, P.; Sbraccia, P.; Goldfine, I.D.; Vigneri, R.; Belfiore, A. Insulin receptor activation by IGF-II in breast cancers: Evidence for a new autocrine/paracrine mechanism. Oncogene 1999, 18, 2471-2479. [CrossRef] [PubMed]

38. Bjorner, S.; Rosendahl, A.H.; Simonsson, M.; Markkula, A.; Jirstrom, K.; Borgquist, S.; Rose, C.; Ingvar, C.; Jernstrom, H. Combined and individual tumor-specific expression of insulin-like growth factor-I receptor, insulin receptor and phospho-insulin-like growth factor-I receptor/insulin receptor in primary breast cancer: Implications for prognosis in different treatment groups. Oncotarget 2017, 8, 9093-9107. [CrossRef]

39. Pan, F.; Hong, L.Q. Insulin promotes proliferation and migration of breast cancer cells through the extracellular regulated kinase pathway. Asian Pac. J. Cancer Prev. 2014, 15, 6349-6352. [CrossRef] 
40. Fox, E.M.; Kuba, M.G.; Miller, T.W.; Davies, B.R.; Arteaga, C.L. Autocrine IGF-I/insulin receptor axis compensates for inhibition of AKT in ER-positive breast cancer cells with resistance to estrogen deprivation. Breast Cancer Res. 2013, 15, R55. [CrossRef]

41. Belfiore, A.; Malaguarnera, R. Insulin receptor and cancer. Endocr. Relat. Cancer 2011, 18, R125-R147. [CrossRef]

42. Cianfrocca, M.; Goldstein, L.J. Prognostic and predictive factors in early-stage breast cancer. Oncologist 2004, 9, 606-616. [CrossRef]

43. Mulligan, A.M.; O’Malley, F.P.; Ennis, M.; Fantus, I.G.; Goodwin, P.J. Insulin receptor is an independent predictor of a favorable outcome in early stage breast cancer. Breast Cancer Res. Treat. 2007, 106, 39-47. [CrossRef] [PubMed]

44. Aljada, A.; Saleh, A.M.; Al-Aqeel, S.M.; Shamsa, H.B.; Al-Bawab, A.; Al Dubayee, M.; Ahmed, A.A. Quantification of insulin receptor mRNA splice variants as a diagnostic tumor marker in breast cancer. Cancer Biomark.: Sect. Dis. Markers 2015, 15, 653-661. [CrossRef] [PubMed]

45. Sierra, J.R.; Tsao, M.S. c-MET as a potential therapeutic target and biomarker in cancer. Adv. Med Oncol. 2011, 3, S21-S35. [CrossRef] [PubMed]

46. Lawrence, R.E.; Salgia, R. MET molecular mechanisms and therapies in lung cancer. Cell Adhes. Migr. 2010, 4, 146-152. [CrossRef] [PubMed]

47. Mo, H.N.; Liu, P. Targeting MET in cancer therapy. Chronic Dis. Transl. Med. 2017, 3, 148-153. [CrossRef] [PubMed]

48. Jia, L.; Yang, X.; Tian, W.; Guo, S.; Huang, W.; Zhao, W. Increased Expression of c-Met is Associated with Chemotherapy-Resistant Breast Cancer and Poor Clinical Outcome. Med Sci. Monit. Int. Med. J. Exp. Clin. Res. 2018, 24, 8239-8249. [CrossRef]

49. Zhang, Y.; Xia, M.; Jin, K.; Wang, S.; Wei, H.; Fan, C.; Wu, Y.; Li, X.; Li, X.; Li, G.; et al. Function of the c-Met receptor tyrosine kinase in carcinogenesis and associated therapeutic opportunities. Mol. Cancer 2018, 17, 45. [CrossRef]

50. Ho-Yen, C.M.; Jones, J.L.; Kermorgant, S. The clinical and functional significance of c-Met in breast cancer: A review. Breast Cancer Res. 2015, 17, 52. [CrossRef]

51. Wang, M.; Liang, L.; Lei, X.; Multani, A.; Meric-Bernstam, F.; Tripathy, D.; Wu, Y.; Chen, H.; Zhang, H. Evaluation of cMET aberration by immunohistochemistry and fluorescence in situ hybridization (FISH) in triple negative breast cancers. Ann. Diagn. Pathol. 2018, 35, 69-76. [CrossRef]

52. Lengyel, E.; Prechtel, D.; Resau, J.H.; Gauger, K.; Welk, A.; Lindemann, K.; Salanti, G.; Richter, T.; Knudsen, B.; Vande Woude, G.F.; et al. C-Met overexpression in node-positive breast cancer identifies patients with poor clinical outcome independent of Her2/neu. Int. J. Cancer 2005, 113, 678-682. [CrossRef]

53. Nakopoulou, L.; Gakiopoulou, H.; Keramopoulos, A.; Giannopoulou, I.; Athanassiadou, P.; Mavrommatis, J.; Davaris, P.S. c-met tyrosine kinase receptor expression is associated with abnormal beta-catenin expression and favourable prognostic factors in invasive breast carcinoma. Histopathology 2000, 36, 313-325. [CrossRef] [PubMed]

54. Baccelli, I.; Stenzinger, A.; Vogel, V.; Pfitzner, B.M.; Klein, C.; Wallwiener, M.; Scharpff, M.; Saini, M.; Holland-Letz, T.; Sinn, H.P.; et al. Co-expression of MET and CD47 is a novel prognosticator for survival of luminal breast cancer patients. Oncotarget 2014, 5, 8147-8160. [CrossRef] [PubMed]

55. Zagouri, F.; Bago-Horvath, Z.; Rossler, F.; Brandstetter, A.; Bartsch, R.; Papadimitriou, C.A.; Dimitrakakis, C.; Tsigginou, A.; Papaspyrou, I.; Giannos, A.; et al. High MET expression is an adverse prognostic factor in patients with triple-negative breast cancer. Br. J. Cancer 2013, 108, 1100-1105. [CrossRef] [PubMed]

56. Yan, S.; Jiao, X.; Zou, H.; Li, K. Prognostic significance of c-Met in breast cancer: A meta-analysis of 6010 cases. Diagn. Pathol. 2015, 10, 62. [CrossRef]

57. Litzenburger, B.C.; Creighton, C.J.; Tsimelzon, A.; Chan, B.T.; Hilsenbeck, S.G.; Wang, T.; Carboni, J.M.; Gottardis, M.M.; Huang, F.; Chang, J.C.; et al. High IGF-IR activity in triple-negative breast cancer cell lines and tumorgrafts correlates with sensitivity to anti-IGF-IR therapy. Clin. Cancer Res. 2011, 17, 2314-2327. [CrossRef]

58. Schwartz, G.K.; Dickson, M.A.; LoRusso, P.M.; Sausville, E.A.; Maekawa, Y.; Watanabe, Y.; Kashima, N.; Nakashima, D.; Akinaga, S. Preclinical and first-in-human phase I studies of KW-2450, an oral tyrosine kinase inhibitor with insulin-like growth factor receptor-1/insulin receptor selectivity. Cancer Sci. 2016, 107, 499-506. [CrossRef] 
59. Hou, X.; Huang, F.; Macedo, L.F.; Harrington, S.C.; Reeves, K.A.; Greer, A.; Finckenstein, F.G.; Brodie, A.; Gottardis, M.M.; Carboni, J.M.; et al. Dual IGF-1R/InsR inhibitor BMS-754807 synergizes with hormonal agents in treatment of estrogen-dependent breast cancer. Cancer Res. 2011, 71, 7597-7607. [CrossRef]

60. Chan, J.Y.; Hackel, B.J.; Yee, D. Targeting Insulin Receptor in Breast Cancer Using Small Engineered Protein Scaffolds. Mol. Cancer Ther. 2017, 16, 1324-1334. [CrossRef]

61. Chia, S.K.; Ellard, S.L.; Mates, M.; Welch, S.; Mihalcioiu, C.; Miller, W.H., Jr.; Gelmon, K.; Lohrisch, C.; Kumar, V.; Taylor, S.; et al. A phase-I study of lapatinib in combination with foretinib, a c-MET, AXL and vascular endothelial growth factor receptor inhibitor, in human epidermal growth factor receptor 2 (HER-2)-positive metastatic breast cancer. Breast Cancer Res. 2017, 19, 54. [CrossRef]

62. Tolaney, S.M.; Ziehr, D.R.; Guo, H.; Ng, M.R.; Barry, W.T.; Higgins, M.J.; Isakoff, S.J.; Brock, J.E.; Ivanova, E.V.; Paweletz, C.P.; et al. Phase II and Biomarker Study of Cabozantinib in Metastatic Triple-Negative Breast Cancer Patients. Oncologist 2017, 22, 25-32. [CrossRef]

(C) 2020 by the authors. Licensee MDPI, Basel, Switzerland. This article is an open access article distributed under the terms and conditions of the Creative Commons Attribution (CC BY) license (http://creativecommons.org/licenses/by/4.0/). 